DIVISION OF THE HUMANITIES AND SOCIAL SCIENCES CALIFORNIA INSTITUTE OF TECHNOLOGY

PASADENA, CALIFORNIA 91125

LANDSCAPE FORMATION IN A SPATIAL VOTING MODEL

Scott E. Page

California Institute of Technology

Ken Kollman

University of Michigan

John H. Miller

Carnegie Mellon University

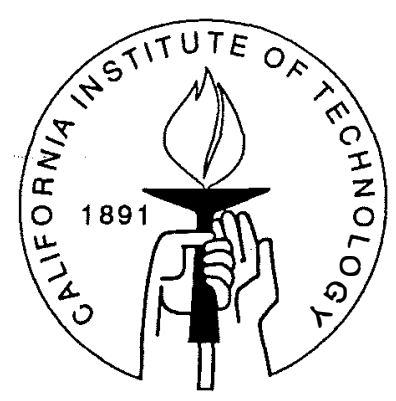

SOCIAL SCIENCE WORKING PAPER 903

August 1994 


\title{
LANDSCAPE FORMATION IN A SPATIAL VOTING MODEL
}

\author{
Scott E. Page Ken Kollman John H. Miller*
}

\section{Introduction}

This paper contributes to the recent literature using complex adaptive systems in the social sciences (Marimon, McGrattan, and Sargent, 1990; Holland and Miller, 1991) by generating hypotheses based on the environment in which dynamic adaptive behavior occurs in a two-party spatial model of elections. The main result of this paper establishes a relationship between voters' preferences and the slope of the electoral landscape on which political parties adapt. Combining this result with an assumption that more gradual slopes make for slower local adaptation (given imperfect, local information), we formulate hypotheses relating voters' preferences and the separation, or divergence, of party platforms. In this paper, we lend support to these hypotheses through computational experiments. In a related paper, which we summarize here, we also find empirical support for these hypotheses. (Kollman, Miller, and Page, 1994).

This analysis fits within a larger research agenda concerning dynamic models of adaptive parties. The research has led to three conclusions: first, that over a series of elections adaptive parties will choose moderate platforms with some degree of separation on issues (Kollman, Miller, and Page, 1992; Page, Kollman, and Miller, 1992); second, that incumbents may win re-election because challengers are unable to locate winning platforms; and third, that changes in the distributions of voters' preferences may influence electoral competition and, consequently, electoral outcomes (Kollman, Miller, and Page, 1993). These findings are noteworthy not simply because they differ from past theoretical results but also because they tend to agree with common wisdom and empirical regularities: parties in two-party systems advocate moderate but distinct platforms, incumbents can

${ }^{*}$ An early version of this paper was presented at the annual meetings of the American Political Science Association, Washington D.C. September 2-5 1993. The authors wish to thank Paul Fischbeck and Joe Oppenheimer for their comments on that version. The Center for Mathematical Studies in Economics and Management Science at Northwestern University and Sun Microsystems are gratefully acknowledged for their support. A portion of this work was funded by NSF grants SBR 94-09602, SBR 94-11025, and SBR 94- 10948 
be hard to defeat, and some electorates are more difficult for candidates to understand than others.

The purpose of this paper is to develop theoretical underpinnings for a general model relating voters' preference distributions and the behavior of adaptive parties in spatial elections. Specifically, we relate changes in party platforms (in response to changes in vote totals) to the distribution of voters' issue strengths, where strength refers to the degree to which a voter cares about an issue. In doing so, we draw a distinction between the actual vote total that a platform receives and its expected vote total based on polling information. To simplify the presentation, we incorporate the metaphor of an electoral landscape upon which parties move. A platform's popularity can be interpreted as an elevation, and parties search for points of high elevation. ${ }^{1}$ A party's ability to locate winning platforms depends upon its adaptive environment: smooth landscapes with steep slopes are climbed more easily than rugged landscapes with gradual slopes.

In the main results of this paper we characterize the slope of the electoral landscape as a function of the distribution of voters' preferences. We find, for example, that voters with extremist preferences form electoral landscapes with more gradual slopes than do voters with centrist preferences. This agrees with our earlier computational findings that adaptive parties facing voters with extremist preferences tended to converge to moderate platforms less quickly than parties facing voters with centrist preferences (Kollman, Miller, and Page, 1993) and with recent empirical work on platform convergence (Kollman, Miller, and Page, 1994).

The remainder is organized as follows: In section 2, we provide an overview of the literature and motivate the use of computational models in the social sciences. In section 3 , we present a basic spatial model of two-party electoral competition and describe the search rules for three types of adaptive parties. In section 4, we discuss the formation of landscapes, formalize the notions of actual and adaptive landscapes, and measure the probability of obtaining an incorrect poll. Section 5 contains the main results. We demonstrate a relationship between classes of voters' preferences and landscape characteristics. In section 6 , we corroborate these analytic results with computational experiments and summarize empirical findings that also lend support to the hypotheses generated by the formal model. The discussion at the end of this paper provides additional intuition about our results and discusses potential applications of these techniques.

\section{Background}

In his seminal work in the spatial theory of elections, Downs (1957) assumes that voters and parties are perfectly informed, parties respond optimally to voters' preferences, and all citizens participate. Downs demonstrates that if preferences can be characterized by distances along a single dimension, then parties in two-party systems converge to the

\footnotetext{
${ }^{1}$ If only parties in real life searched for the high ground!
} 
median voter's ideal point. That is, the parties will take virtually identical ideological stands. The vast literature on spatial elections subsequent to Downs has extended his original model to allow for, among other features, multiple issue dimensions, probabilistic voting, mixed strategies, voter abstention, and multiple parties. In these extended models, Downs' basic result holds only under restrictive conditions (Plott, 1967).

These spatial models of elections also rely on the assumption that voters and candidates have perfect information and computational ability. Even models with imperfect information assume that candidates or parties know perfectly the probabilities of others' actions or states of the world. Though few positive theorists believe that voters and candidates solve problems of such complexity, they accept rational models as benchmarks for human and organizational behavior.

Theories of elections that explicitly incorporate empirical research have relaxed strict informational and computational assumptions for voters. According to one set of theories, voting behavior is a product of sociological predispositions (Campbell, et al., 1960; Keith, et al., 1992). For example, many studies of voting behavior assume, or conclude, that voters derive their opinions about politics and voting decisions from symbols and personal loyalties (Sears, et al., 1979). Candidates, knowing this, try to highlight certain characteristics about themselves or their opponents for the purpose of exploiting voters' psychological attachments to party symbols, racial groups, or ideologies. Popular discussions about the Willie Horton advertisements of the 1988 campaign assume this type of decision-making.

Alternative theories state that voting behavior is based on voters' perceptions and experiences of the performance of the economy (Fiorina, 1980; Kramer, 1972). Voters need to know little more than their economic situation and the health of the general economy to vote informatively. In each of these theories, voters' behavior is rationally based, but it is assumed that voters rely on low-information cues to guide them (Popkin, 1992).

Among social scientists there is little doubt that voters have biases and possess incomplete information, and that these deviations from rational behavior influence electoral politics. The starting point for a theory of adaptive party behavior is to assume similar limitations, but for political parties. An adaptive political party differs from an optimizing political party in its access to information, its methods of processing information, and its ability to reposition itself ideologically based on new information. In our formulation, adaptive parties gather information by commissioning polls of the electorate. They neither know, nor act as if they know, the utility functions of every voter. Our adaptive parties rely on heuristics (rules of thumb) to process this information and appeal to voters. Finally, maneuvering in a spatial environment, adaptive parties make only local adaptations. They may not scan the entire space and instantly locate at the optimal platform.

Our approach represents a significant but natural departure from the literature in that it models parties as adaptive and part of a dynamic complex system rather than as 
optimizers in a static framework. There are many benefits to using a complex adaptive systems approach to social science ${ }^{2}$. First, these models allow researchers to explore the relationship between optimization and adaptation. A common criticism of attempts to relax the rationality assumption is that any single behavioral assumption is ad hoc. Computational models allow for the testing of a variety of behavioral models and for the discovery of common adaptive strategies. Such flexibility enables one to retain a degree of agnosticism as to exactly how adaptive parties respond to information. For instance, we need not be wedded to one decision-making rule be it rational choice or random search. Second, these models enable the testing of multiple hypotheses about the underlying adaptive environment. This is possible because any state of the system is fully recoverable. Third, computational models retain a level of logical consistency enforced by the computer language, yet they allow tremendous flexibility as described above. Finally, these models can be used to test the robustness of theoretical models by relaxing sets of assumptions.

In the context of the theory of democratic elections, computational models are complementary to both theoretical and empirical studies. Computational modeling can bridge theory building and testing-we note that the present formal analysis would never have been undertaken had it not been for insights that arose from earlier computational investigations. In this paper, we demonstrate how a theoretical analysis of landscape formation explains qualitative computational findings. Furthermore, computational modeling can be used to identify testable hypotheses - in this case, about how the distribution of voters' preferences may effect rates of party convergence.

\section{A Model of Adaptive Parties}

In this section we describe a basic spatial model and the heuristics employed by our adaptive parties. The section is divided into three parts. First, we present a spatial model in which preferences are determined by both ideal points and strengths on issues. Second, we describe an election sequence. Finally we define the search rules used by the adaptive parties. ${ }^{3}$

\subsection{A Basic Spatial Model}

We assume that each voter attaches both a strength and an ideal position to each of $n$ issues. Voter $j$ 's strength on issue $i, s_{j i} \subset \Re^{+}$, measures the issue's relative importance to the voter. A voter considers an issue of strength zero irrelevant. The ideal position of voter $j$ on issue $i, x_{j i} \subset \Re$, denotes the voter's preferred position on the issue. The utility to voter $j$ from a party's platform, $y \in \Re^{n}$, equals the negative of the squared weighted

\footnotetext{
${ }^{2}$ See Holland and Miller, 1991, for a more complete discussion of this type of approach. Many of the arguments lifted below are excerpted from their paper.

${ }^{3}$ For a more complete description, see Kollman, Miller, and Page, 1992.
} 
Euclidean distance between the vector of $j$ 's ideal positions and the party's platform, weighted by the voter's strengths:

$$
u_{j}(y)=-\sum_{i=1}^{n} s_{j i} \cdot\left(x_{j i}-y_{i}\right)^{2} .
$$

We make the standard assumptions that voter $j$ casts a ballot for the party whose platform yields the higher utility. We further assume that parties are purely office seeking. ${ }^{4}$

We want to compare how variations in the preference distribution effect adaptive party competition. We consider three types of preferences:

- Extremist: Voters place more weight on issues on which they have extreme views, e.g. abortion and gun control.

- Centrist: Voters place more weight on issues on which they have centrist views, e.g. foreign policy and social security.

- Independent: Voters' weights on issues are statistically independent of their preferred positions.

These notions are formalized in section 5 .

\subsection{The Sequence of Elections}

An electoral sequence begins with the creation of two randomly generated, initial party platforms. One party is arbitrarily chosen to be the incumbent, whose platform remains fixed during the first election. Prior to the first election, there is a campaign in which the challenger adapts a new platform. The challenger party gathers information by undertaking polls of the electorate and alters its platform in response to the polling data. Polls provide a noisy signal of the popularity of proposed platforms. The length of a campaign equals the number of polls commissioned by the challenger party. At the completion of the campaign, the challenger party selects a platform and the two parties stand for election with the winning party becoming the fixed incumbent.

The losing party then becomes the challenger, and a new campaign .ensues culminating in another election. At the completion of each sequence of elections, we measure the separation of party platforms, the clistances of winning platforms from the party's initial platform, and the total utility to the voters of the winning platform. We also monitor the percentage of the time that the challenger party wins the election. Adaptive parties suffer from limited information and bounded computational abilities, as such they often fail to locate winning platforms during their finite campaigns.

\footnotetext{
${ }^{4}$ Kollman, Miller, and Page, 1992, addresses adaptive parties with preferences over policies as well.
} 


\subsection{Search Techniques}

For reasons mentioned in section 2, we consider various types of adaptive search rules. Three algorithms were used: a genetic algorithm, multi-step hillclimbing, and random search. Each of the algorithms satisfies two criteria. First, each has been used extensively in nonlinear search, so we have some understanding of its strengths and weaknesses. Second, each corresponds to a plausible description of how parties may actually select candidates and platforms.

The genetic algorithm (GA) is by far the most complicated of the three. ${ }^{5}$ A GA begins with a randomly generated population of platforms, in our case twenty platforms, and creates new populations by a process that mimics natural evolution. Let $p_{0}$ be the initial population of strings. In our setting, these can be thought of as either separate candidates or various platforms for a. mutable candidate. Platforms in the population are reproduced based on their relative performances. For example, a platform that fairs poorly against the incumbent is not as likely to be reproduced as a platform that defeats the incumbent. In keeping with the biological metaphor, this can be thought of as "survival of the fittest." 6

Two genetic operators are then applied to the platforms that survive reproduction: crossover and mutation. The crossover operator pairs platforms and then exchanges their positions on a random set of issues. We might think of political candidates borrowing ideas from one another during the primary season, or of factions on a platform committee adopting positions from other proposals. Finally, the mutation operator allows for minor platform changes. At the completion of reproduction, crossover, and mutation, the population $p_{1}$ is completed and the GA is iterated. Each application of the GA is referred to as a generation. The number of generations corresponds to the length of the campaign.

The multi-step hillclimbing algorithm operates on a single platform rather than a population. The algorithm takes the party's last position (in the first election this is the initial platform) as its starting point, $y^{*}$. The algorithm has two steps. First, a new platform $y_{1}$ is randomly created in a neighborhood of $y^{*}$. If the new platform is better, i.e., if it obtains more votes against the incumbent, it becomes $y^{*}$, otherwise $y^{*}$ remains unchanged. This process continues until the end of the campaign. The multistep hillclimbing algorithm is intended to represent parties that use polling data to fine tune their candidate's platform.

The random search algorithm begins by randomly creating a number of platforms in a neighborhood of the party's current platform. The best platform from this set is then selected by the party. The random search algorithm is meant to correspond to parties who choose from among a set of volunteers whose platforms are fixed.

\footnotetext{
${ }^{5}$ See Holland (1975) for the original formulation of the genetic algorithm, or Goldberg (1988) for a more gentle introduction.

${ }^{6}$ After reproduction there may be multiple copies of the better platforms.
} 


\section{Landscapes}

In the first part of this section, we discuss adaptive party performance with respect to landscapes. Then we show how the probability of an incorrect poll depends upon the slope of the landscape.

\subsection{Actual and Adaptive Landscapes}

In our model there are two conceptions of landscapes, actual landscapes and adaptive landscapes. Note that the percentage of the vote received by the challenger party is a function of the preferences of voters and the position of the incumbent party. The actual landscape consists of the percentage of the vote received by the challenger party at each platform, holding everything else constant. If a party is purely office seeking, fully informed, free from computational constraints, and free to locate anywhere in ideological space, then it would choose the platform of highest elevation, i.e., the platform receiving the largest percentage of the vote. Our assumptions that parties have limited information about voter's' preferences, that they respond to that information using hueristic procedures that may not be optimal, and that they can only move locally, may preclude locating the optimal platform. Thus, they face an adaptive landscape characterized by only local and imperfect knowledge about the voters. Whereas the actual landscape consists of the actual vote percentages corresponding to all possible platform choices, the adaptive landscape consists of the perceived vote percentages of the platforms tested by the challenger party through polls.

An adaptive party's knowledge of the landscape is restricted in two ways. First, the vote percentages that parties learn through polling are estimates of the actual percentages. Second, the parties do not have estimates of the elevations of all platforms, but only of those platforms in the neighborhood of their current platform which they have tested.

To illustrate the distinction between the actual and the adaptive landscapes, consider two extreme cases, which we refer to as the Nebraska and Mount Fuji landscapes. Note the topography of Nebraska. Traveling westward across Nebraska's nearly four hundred miles, one might never notice an increase in elevation. In fact, Nebraska rises over fortythree hundred feet from Omaha at its eastern edge to to the western gate at Johnson township in Kimball County. Suppose that two mountain climbers are placed randomly in Nebraska and told to locate. Nebraska's point of highest elevation. Suppose further that the first climber is outfitted with a precise map of Nebraska's topography and a compass and that the second climber is provided with neither. For the first climber, who has perfect, global information, the task is accomplished easily. For the second climber, whose information is both local and imperfect, the task would be more difficult; the Nebraska landscape looks roughly the same everywhere. This climber might be tempted to choose the nearest hill, or mound, presuming one exists, or may wander aimlessly. 
Note that in the scenario just described, the adaptive landscape creates problems for the climber as a result of both the local and imperfect nature of the climber's information. If a climber possessed global, though imperfect information, i.e., if she perceived slightly inaccurate elevations over the entire state, then she would recognize the general elevation difference and move westward. If a climber possessed local, but perfect information, she would notice Nebraska's gentle tilt and adapt westward as long as she could see beyond small local knolls.

At the other extreme lies the Mount Fuji landscape, where a single steeply sloped peak dominates the topography. If the second climber were to confront such a landscape, she could easily scale the steep, single-peaked hill even with the information limitations. Neither restriction would prevent her from acting "as if" she had perfect, global information and were optimizing.

These examples suggest that the slope of the actual landscape effects the performance of adaptive parties, whose information is both imperfect and local. More generally, as we discuss at the end of the paper, this sort of result may hold in other interesting settings.

\subsection{Incorrect Polls}

We can formalize the idea that more subtle slopes slow the rate of adaptation by applying elementary statistics to the problem of measuring the difference between proportions of populations. Suppose that a party's current platform receives a proportion of the vote equal to $p$, and that the platform it is considering receives a proportion of the vote equal to $q$. Suppose further that $q>p$, i.e., that the new platform leads to a higher proportion of the vote. Let $p^{s}$ and $q^{s}$ be the sample proportions from simple random samples of the voters of size $n$. We are interested in how often the party receives polling data with an incorrect signal, namely $q^{s}<p^{s}$. The point estimator of concern is $p^{s}-q^{s}$, which is approximately normally distributed. Its standard deviation is given by:

$$
\sigma_{p^{s}-q^{s}}=\sqrt{\frac{p(1-p)}{n}+\frac{q(1-q)}{n}} .
$$

The number of standard deviations away from the mean, is given by:

$$
z=\frac{p-q}{\sigma_{p^{s}-q^{s}}}
$$

The probability that a poll is misleading equals the probability that the mean of a standard normal distribution is less than $z$. Most electoral polls consult between six-hundred and two-thousand voters. For the purposes of explanation, suppose that $n=800$ and that $p$ and $q$ are both near 0.5 . A simple calculation shows that $\sigma_{p^{s}-q^{s}}$ is approximately equal to 0.025 , or $2.5 \%$. A platform adaptation which increased a party's 
vote total by one-half a percent could easily be misinterpreted. The probability that a random poll would show a decrease in vote total equals the probability of being at least one-fifth of a standard deviation below zero in a standard normal distribution which is approximately 0.42 . This example shows that slight differences in vote totals may be difficult for parties to recognize.

\section{A Formal Model of Landscape Gradients}

In this section, we present a formal analysis of the gradients of landscapes formed by centrist, extremist, and circular preferences. We show that extremist preferences form landscapes with gradual gradients, that centrist preferences form landscapes with steep gradients, and that circular preferences form landscapes with moderate gradients. These results agree with previous computational findings and with new findings presented in section 5 .

We examine a one dimensional projection onto issue 1. Preferences are characterized by weighted Euclidean distance. The utility of a platform $x$ to a voter with an ideal platform $a$ and a strength vector $s$ is given by

$$
U(x, a)=-\sum_{i=1}^{n} s_{i} \cdot\left(x_{i}-a_{i}\right)^{2} .
$$

We make the following assumption about voters' ideal points:

\section{(A1) Voter ideal points are uniformly distributed on issue 1 on $[-1,1]$}

Given this assumption we can formalize the notions of centrist, extremist, and circular preferences:

$$
\begin{array}{ll}
\text { Centrist Preferences: } & s_{i}=\left(1-\left|a_{i}\right|\right) \\
\text { Extremist Preferences: } & s_{i}=\left|a_{i}\right| \\
\text { Circular Preferences: } & s_{i}=\frac{1}{2}
\end{array}
$$

Note for all types of preferences strengths lie in the interval $[0,1]$. We make two further assumptions about the distribution of voters' ideal points:

(A2) There are infinitely many voters at each ideal point in $[-1,1]$. 
Assumption A2 allows us to take integrals at each point on the line, which simplifies the problem. With smaller numbers of agents at each point, the landscape grows more rugged due to sampling noise. This ruggedness is precisely what we capture by considering adaptive parties.

(A3) For voters at each ideal point in $[-1,1]$ on issue 1 , the utility difference between the challenger and the incumbent on the other $n-1$ issues (denoted by $e)$ is uniformly distributed on $[-b, b]$ where $b \geq 1$.

Assumption A3 appears problematic. Notice that distances on separate issues are being summed. Therefore, a more realistic assumption would be normally distributed differences. Three comments are in order. First, the assumption of normally distributed differences complicates the model to the point of intractability, as we make evident. Second, the voters who switch which candidate they prefer based on candidate movements on issue 1 have utility differences in $(-1,1)$. If the differences are normally distributed with zero mean and high variance, then the uniformity assumption is a reasonable approximation. Third, as discussed in section 5, computational experiments agree with the analytical results, so the assumption does not appear too important.

\subsection{Landscape Formation}

We now want to calculate the vote total that the challenger party receives as a function of its position on issue $1, x$, the incumbent's position, $y$, and the divergence of opinion on other issues, parameterized by $b$. The challenger's vote total equals the measure of the agents whose votes he receives. This vote measure depends upon whether the challenger's position on issue $1, x$, lies in the upper, $U$, middle, $M$, or lower, $L$, part of the interval. See the appendix for a formal characterization of preferences.

Claim 5.1 Given $y \geq 0$, the measure of votes that the challenger party located at $x$ receives against an incumbent located at $y$ if voters have centrist preferences equals

$$
c(x, y, b)=\frac{6 b-3 x^{2}+\operatorname{sign}(x) \cdot x^{3}+3 y^{2}+y^{3}}{12 b}
$$

pf: see appendix.

Therefore the slope of the centrist landscape on issue 1 with respect to the challenger's position is given by: 


\section{Corollary 5.1}

$$
\frac{\partial c(x, y, b)}{\partial x}=\frac{\operatorname{sign}(x) \cdot x^{2}-2 x}{4 b} \quad x \in[-1,1]
$$

pf: follows directly.

We want to compare this slope to the slopes of landscapes formed by extremist and circular preferences.

Claim 5.2 Given $y \geq 0$, the measure of votes that the challenger party located at $x$ receives against an incumbent located at $y$ if voters have extremist preferences equals

$$
\epsilon(x, y, b)=\frac{6 b-\operatorname{sign}(x) \cdot x^{3}-y^{3}}{12 b}
$$

pf: see appendix.

Corollary 5.2 The slope of the extremist landscape on issue 1 with respect to the challenger's position is given by:

$$
\frac{\partial e(x, y, b)}{\partial x}=\frac{-\operatorname{sign}(x) \cdot x^{2}}{4 b} \quad x \in[-1,1]
$$

pf: follows directly.

Finally, we can do the same analysis for circular preferences.

Claim 5.3 Given $y \geq 0$, the measure of votes that the challenger party located at $x$ receives against an incumbent located at $y$ if voters have circular preferences equals

$$
r(x, y, b)=\frac{4 b-x^{2}+y^{2}}{8 b} \quad x \in[-1,1]
$$

pf: omitted. 
Corollary 5.3 The slope of a landscape formed by circular preferences on issue 1 with respect to the challenger's position is given by:

$$
\frac{\partial r(x, y, b)}{\partial x}=\frac{-x}{4 b} \quad x \in[-1,1]
$$

pf: follows directly.

For each type of preferences it is straightforward to show that the measure of votes the challenger receives is maximized at $x=0$, the median position on issue 1 . The votes the challenger receives as a function of the challenger's position on issue 1 are shown in Figure 1.

\subsection{Landscape Comparison}

In this section we make formal claims about the slopes of the landscapes within and across preference distributions.

Claim 5.4 For all three types of prejerences the slope is independent of the opponent's position on issue 1.

pf:

$$
\frac{\partial^{2} r(x, y, b)}{\partial x \partial y}=\frac{\partial^{2} e(x, y, b)}{\partial x \partial y}=\frac{\partial^{2} c(x, y, b)}{\partial x \partial y}=0
$$

However, changes in the opponents positions do shift landscapes vertically. See Figure 2.

Claim 5.5 For all three types of preferences the slope is decreasing in $b$.

pf: Consider the case of extremist preferences,

$$
\frac{\partial e(x, y, b)}{\partial b}=\frac{-e(x, y, b)}{b}
$$

Since $e(x, y, b) \geq 0$. The other cases are proved similarly. See Figure 3 . 
Claim 5.6 For any $(x, y, b)$ with $|x|>0$ the slope of a landscape formed by centrist preferences is strictly steeper than the slope of a landscape formed by extremist preferences.

pf: Suffices to show

$$
\left|\frac{\partial c(x, y, b)}{\partial x}\right|>\left|\frac{\partial r(x, y, b)}{\partial x}\right| \quad x \in[1,1], x \neq 0
$$

By symmetry the inequality can be rewritten

$$
\frac{-2 x-x^{2}}{4 b}>\frac{x^{2}}{4 b} \quad x \in[-1,0)
$$

which reduces to

$$
-x^{2}>x \quad x \in[-1,0)
$$

which completes the proof.

Claim 5.7 For any $(x, y, b)$ with $|x|>0$ the slope of a landscape formed by centrist preferences is strictly steeper than the slope of a landscape formed by circular preferences.

pf: Suffices to show

$$
\left|\frac{\partial r(x, y, b)}{\partial x}\right|>\left|\frac{\partial e(x, y, b)}{\partial x}\right| \quad x \in[-1,1], x \neq 0
$$

By symmetry the inequality can be rewritten

$$
\frac{-2 x-x^{2}}{4 b}>\frac{-x}{4 b} \quad x \in[-1,0)
$$

which reduces to

$$
-x^{2}>x \quad x \in[-1,0)
$$

which completes the proof. 
Claim 5.8 For any $(x, y, b)$ with $|x|>0$ the slope of a landscape formed by circular preferences is strictly steeper than the slope of a landscape formed by extremist preferences.

pf: Suffices to show

$$
\left|\frac{\partial r(x, y, b)}{\partial x}\right|>\left|\frac{\partial e(x, y, b)}{\partial x}\right| \quad \sin [-1,1], x \neq 0
$$

By symmetry the inequality can be rewritten

$$
\frac{-x}{4 b}>\frac{x^{2}}{4 b} \quad x \in[-1,0)
$$

which reduces to

$$
-x>x^{2} \quad x \in[-1,0)
$$

which completes the proof.

To summarize these claims, voters with centrist preferences form landscapes with steeper slopes than do voters with circular preferences, and voters with circular preferences form landscapes with steeper slopes than voters with extremist preferences. These claims establish a direct relationship between the distribution of voters' strengths and the slope of electoral landscapes.

\section{Computational Experiments and Empirical Evi- dence}

This section presents findings from computational experiments similar to those performed in Kollman, Miller, and Page (1993). In addition, we summarize empirical findings which also corroborate our hypotheses. The following parameters were used. The electoral environment consisted of ten issues. There were 2501 voters in the population and the the polls sampled randomly drawn subpopulations of size $251{ }^{7}$

\footnotetext{
${ }^{7}$ Similar numbers were found with many other sets of parameters. Qualitatively, the results which we report appear to hold irrespective of the particular parameter values or the choice of search heuristic. Numbers from computational experiments with parties using random search and genetic algorithms look similar. The only experiments in which our results did not hold significantly occurred when the number of adaptations per elections substantially exceeds the number of issues. For example, if a genetic algorithm is run for five hundred iterations with only one issue then the differences are not significant. We would argue that with so many adaptations that the party might be more accurately thought of as fully-informed and optimizing.
} 
The correlations between strengths and ideal positions on issues were chosen exactly as described in section 5. In Table 1 we present numerical summaries from one hundred sets of ten simulated elections with multi-step hill climbing parties which performed forty adaptations per elections.

In the numbers presented here we focus solely on platform convergence. However, in computations not shown, we found that the slopes of the landscapes differed significantly in the expected directions: centrist preferences formed the steepest sloped landscapes. To capture the amount of convergence we rely on two measures: distance to the median and platform separation. These numbers are shown in Table 1. Distance to the median equals the distance of the incumbent's platform to the platform consisting of the median on each individual issue. Table 2 shows the results and difference of means tests which find strong support for our hypotheses. We find that parties are furthest from the median when the voters are extremist on every issue and nearest when the voters are centrist.

To account for the possibility that the parties may be converging to a platform $p$ different from the median platform and that what we are capturing is the distance from $p$ to the median, we also measure platform separation. Platform separation equals the Euclidean distance between the incumbent and the challenger's platform at the completion of an election. These numbers are shown in Tables 1 and 3 and further support our hypotheses. Together the tests show that parties are both furthest apart and furthest from the median when voters preferences are extremist. Also, as expected, parties are nearest together and closest to the median when voters are centrist.

The tests just described consider environments in which voter preferences on every issue are of one type; they might all be centrist, for example. We might also wonder what happens when on some issues voters' preferences are centrist, on others preferences are circular, and still on others preferences are extremist. Do we see similar phenomena within a single election? Will adaptive parties adapt similar positions on centrist issues and distinct positions on extremist issues? Table 4 presents numerical summaries from computational experiments in which there was no correlation between strengths and preferences except for on issues one and two. Agents had centrist preferences on issue one and extremist preferences on issue two. The numbers show significant differences in the separation of parties' platforms on these issues in the expected direction: the parties are closer together on issue one, the centrist issue, than on issue two.

These last computations are especially relevant in considering empirical tests of the model. Actual voter preferences will differ in their degree of extremism across issues. The relevant question is whether an issue's degree of extremism is correlated with the separation of the parties on the issue. In a related paper, we test this prediction using American National Election Study data on party convergence in the United States (Kollman, Miller, and Page, 1994). In our specification, a voter's preference on an issue becomes more extreme as the strength they attach to the issue increases with their ideal point's distance from the median voter's ideal point. We employ an OLS model and find a significant relationship between the level of extremism and the perceived separation of party platforms. The empirical result, though by no means conclusive, lends strong 
support to our model and gives reason to be optimistic about the use of computational models to identify and explain behavioral regularities.

\section{Discussion}

Our theoretical results suggest that the strength voters attach to issues, if related to voters' positions on issues, can infuence how political parties campaign for elective office. An adaptive party may have difficulty finding successful campaign messages if the electorate contains a substantial percentage of extremist voters. The slope of the electoral landscape may partially explain why some candidates in two-party systems remain extremist, even though they might fare better with voters if their messages became moderate. Electoral landscape characteristics may also help explain why candidates sometimes "grope about" searching for the right message to appeal to voters rather than immediately deciding upon a policy prescription.

In this paper, we have advanced the notion of parties searching electoral landscapes and suggested that landscape characterists influence adaptive search and, therefore, policy outcomes. The main result of this paper has been to establish a formal relationship between voters' preferences and the slope of the electoral landscape. We hypothesized that more gradual landscape slopes hinder adaptation, resulting in less responsive parties. Finally, we corroborated this hypothesis with computational work.

In future work it is worth exploring whether the inability of imperfectly and locally informed agents to recognize gradual slopes has broader application in the social sciences. Applications of this idea may be possible in a variety of fields. For example, in labor economics, income levels depend upon workers' attributes. Attribute space together with expected income form a career landscape upon which agents can adapt. Would one find, for example, that along some dimensions the slope is more gradual? Or perhaps the landscape is rugged? And would one find the under-accumulation of those attributes? ${ }^{8}$ Product differentiation models also appear to be a natural application. The analysis of adaptive behavior will be greatly facilitated by the interaction of mathematical, computational, and empirical studies.

\footnotetext{
${ }^{8}$ Thanks to Daniel Vincent for suggesting this example.
} 


\section{Table 1}

\section{Circular Preferences}

\begin{tabular}{|c|c|c|c|}
\hline election & \% win & dist winner & separation \\
\hline 1 & $0.82(0.38)$ & $108.90(25.82)$ & $50.15(20.36)$ \\
2 & $0.45(0.50)$ & $108.12(24.83)$ & $40.64(18.43)$ \\
3 & $0.57(0.50)$ & $102.62(21.84)$ & $33.47(15.79)$ \\
4 & $0.57(0.50)$ & $99.87(19.07)$ & $27.58(14.33)$ \\
5 & $0.50(0.50)$ & $98.51(17.64)$ & $23.47(12.90)$ \\
6 & $0.47(0.50)$ & $96.79(15.37)$ & $20.23(12.03)$ \\
7 & $0.46(0.50)$ & $95.13(14.54)$ & $17.05(10.45)$ \\
8 & $0.43(0.50)$ & $94.98(13.52)$ & $14.63(9.21)$ \\
9 & $0.40(0.49)$ & $94.83(12.26)$ & $12.78(8.53)$ \\
10 & $0.36(0.48)$ & $94.39(11.75)$ & $11.47(7.80)$ \\
11 & $0.33(0.47)$ & $93.78(11.25)$ & $10.53(7.17)$ \\
12 & $0.30(0.46)$ & $93.53(10.33)$ & $9.92(7.01)$ \\
\hline
\end{tabular}

2501 voters, 251 voters in each poll, 10 issues, 500 trials

\section{Centrist Preferences}

\begin{tabular}{|c|c|c|c|}
\hline election & \% win & dist winner & separation \\
\hline 1 & $0.90(0.29)$ & $106.78(26.23)$ & $45.55(18.63)$ \\
2 & $0.51(0.50)$ & $103.99(22.84)$ & $34.26(15.73)$ \\
3 & $0.66(0.47)$ & $100.72(20.88)$ & $25.93(13.08)$ \\
4 & $0.66(0.48)$ & $98.89(18.13)$ & $19.99(11.24)$ \\
5 & $0.64(0.48)$ & $96.30(15.87)$ & $15.45(9.24)$ \\
6 & $0.59(0.49)$ & $94.30(14.27)$ & $12.24(8.01)$ \\
7 & $0.54(0.50)$ & $92.96(11.77)$ & $9.76(6.65)$ \\
8 & $0.47(0.50)$ & $93.05(10.44)$ & $8.02(5.75)$ \\
9 & $0.46(0.50)$ & $91.30(8.87)$ & $6.75(4.83)$ \\
10 & $0.37(0.48)$ & $92.08(8.50)$ & $5.79(4.43)$ \\
11 & $0.37(0.48)$ & $91.66(7.97)$ & $4.97(4.02)$ \\
12 & $0.32(0.47)$ & $91.38(7.18)$ & $4.49(3.45)$ \\
\hline
\end{tabular}

2501 voters, 251 voters in each poll, 10 issues, 500 trials

Standard errors of the 500 data points are given in parenthesis. To obtain standard errors of the means, we must divide by $\sqrt{500}$ 


\section{Extremist Preferences}

\begin{tabular}{|c|c|c|c|}
\hline election & \% win & dist winner & separation \\
\hline 1 & $0.73(0.44)$ & $113.48(30.20)$ & $51.44(20.48)$ \\
2 & $0.38(0.48)$ & $110.01(28.05)$ & $44.26(19.64)$ \\
3 & $0.45(0.50)$ & $107.02(25.81)$ & $39.65(18.00)$ \\
4 & $0.44(0.50)$ & $104.39(24.72)$ & $35.50(16.60)$ \\
5 & $0.43(0.49)$ & $101.49(22.55)$ & $32.34(16.09)$ \\
6 & $0.35(0.48)$ & $101.16(21.23)$ & $30.11(15.23)$ \\
7 & $0.34(0.47)$ & $99.96(20.53)$ & $28.24(14.34)$ \\
8 & $0.31(0.46)$ & $99.21(18.51)$ & $26.32(13.44)$ \\
9 & $0.30(0.46)$ & $98.22(18.36)$ & $24.82(12.71)$ \\
10 & $0.32(0.47)$ & $97.58(17.70)$ & $23.56(12.79)$ \\
11 & $0.26(0.44)$ & $96.54(16.23)$ & $22.43(12.81)$ \\
12 & $0.23(0.42)$ & $95.68(15.52)$ & $21.72(12.72)$ \\
\hline
\end{tabular}

2501 votel's, 251 voter's in each poll, 10 issues, 500 trials

Standard errors of the 500 data points are given in parenthesis. To obtain standard errors of the means, we must divide by $\sqrt{500}$ 


\author{
Table 2 \\ Distance to Median of Winner \\ Multi-Step Hillclimbing Adaptive Parties \\ 200 Trials
}

ELECTION 2

Preference Type

Centrist

Extremist

Circular

Difference

Cent-Circ

Cent-Ext

Circ-Ext
Mean

26.59

30.66

29.50

T-Stats

2.945

4.336

1.185

\section{ELECTION 5}

Preference Type

Centrist

Extremist

Circular

Difference

Cent-Circ

Cent-Ext

Circ-Ext

\section{ELECTION 10}

Preference Type

Centrist

Mean

Extremist

4.71

9.46

Circular

Difference

Cent-Circ

Cent-Ext

7.36

T-Stats

7.552

11.526

4.471 


\author{
Table 3 \\ Platform Separation \\ Multi-Step Hillclimbing Adaptive Parties \\ 200 Trials
}

ELECTION 2

\section{Preference Type}

Centrist

Extremist

Circular

Difference

Cent-Circ

Cent-Ext

Circ-Ext
Mean

64.05

75.05

73.30

T-Stats

3.056

3.581

0.543

\section{ELECTION 5}

Mean

30.35

44.96

38.20

T-Stats

3.929

6.974

2.937

ELECTION 10

Preference Type

Centrist

Extremist

Circular

Difference

Cent-Circ

Cent-Ext

Circ-Ext
Mean

18.36

33.22

25.95

T-Stats

5.303

9.065

4.075 


\section{Table 4}

\section{Party Separation for Centrist vs. Extremist Voters' Distribution}

\begin{tabular}{|c|c|c|c|}
\hline election & \% win & centrist issue & extremist issue \\
\hline 1 & $0.76(0.43)$ & $1.76(1.30)$ & $1.77(1.29)$ \\
2 & $0.44(0.50)$ & $1.63(1.22)$ & $1.60(1.20)$ \\
3 & $0.54(0.50)$ & $1.44(1.11)$ & $1.52(1.10)$ \\
4 & $0.49(0.50)$ & $1.32(1.04)$ & $1.41(1.04)$ \\
5 & $0.49(0.50)$ & $1.19(0.96)$ & $1.33(0.98)$ \\
6 & $0.46(0.50)$ & $1.09(0.89)$ & $1.21(0.97)$ \\
7 & $0.44(0.50)$ & $1.00(0.86)$ & $1.16(0.95)$ \\
8 & $0.38(0.49)$ & $0.94(0.80)$ & $1.10(0.89)$ \\
9 & $0.37(0.48)$ & $0.87(0.79)$ & $1.08(0.87)$ \\
10 & $0.31(0.46)$ & $0.82(0.70)$ & $1.06(0.85)$ \\
11 & $0.31(0.46)$ & $0.78(0.66)$ & $1.04(0.86)$ \\
12 & $0.26(0.44)$ & $0.78(0.65)$ & $1.03(0.85)$ \\
\hline
\end{tabular}

2501 voters, 251 voters in each

poll, 10 issues, 500 trials

Standard errors of the 500 data points are given in parenthesis. To obtain standard errors of the means, we must divide by $\sqrt{500}$ 


\section{A Appendix 2}

\section{A.1 Preferences}

Without loss of generality we assume that the incumbent's position on issue 1 is denoted by $\mathrm{y}$ in $[-1,0]$ and that the challenger's position on issue 1 is denoted by $\mathrm{x}$ in $[-1,1]$. Recall that e denotes the utility difference between the challenger's platform and the incumbent's platform on the other (n-1) issues. We can now fully characterize preferences on issue 1:

\section{Centrist Preferences}

voter at $a \in[-1,1]$

Case $\mathrm{U}: x \in[0,1]$

$$
\text { Utility of Challenger Utility of Incumbent }
$$

$\begin{array}{lll}a \geq x & (1-a)(a-a)+e & (1-a)(a-y) \\ 0 \leq a \leq x & (1-a)(x-a)+e & (1-a)(a-y) \\ y \leq a<0 & (1+a)(x-a)+e & (1+a)(a-y) \\ a \leq y & (1+a)(x-a)+e & (1+a)(y-a)\end{array}$

Case M: $x \in[y, 0]$
$a \geq 0$
$(1-a)(a-x)+e$
$(1-a)(a-y)$
$x \leq a \leq 0$
$(1+a)(a-x)+e$
$(1+a)(a-y)$
$y \leq a \leq x$
$(1+a)(x-a)+e$
$(1+a)(a-y)$
$a \leq y$
$(1+a)(x-a)+e$
$(1+a)(y-a)$

Case L: $x \in[-1, y]$
$a \geq 0$
$(1-a)(a-x)+e$
$(1-a)(a-y)$
$y \leq a \leq 0$
$(1+a)(a-x)+\epsilon$
$(1+a)(a-y)$
$x \leq a \leq y$
$(1+a)(a-x)+e$
$(1+a)(y-a)$
$a \leq x$
$(1+a)(x-a)+e$
$(1+a)(y-a)$ 


\section{Extremist Preferences}

voter at $a \in[-1,1]$

Case U: $x \in[0,1]$

$a \geq x$

$0 \leq a \leq x$

$y \leq a \leq 0$

$a \leq y$

Case M: $x \in[y, 0]$

$a \geq 0$

$x \leq a \leq 0$

$y \leq a \leq x$

$a \leq y$

$$
\begin{aligned}
& a(a-x)+e \\
& -a(a-x)+e \\
& -a(x-a)+e \\
& -a(x-a)+e
\end{aligned}
$$

\section{Utility of Challenger}

$$
\begin{aligned}
& a(a-x)+\epsilon \\
& a(x-a)+\epsilon \\
& -a(x-a)+\epsilon \\
& -a(x-a)+e
\end{aligned}
$$

Utility of Incumbent

$$
\begin{aligned}
& a(a-y) \\
& a(a-y) \\
& -a(a-y) \\
& -a(y-a)
\end{aligned}
$$

Case L: $\mathrm{x} \in[-1, y]$
$a \geq 0$
$y \leq a \leq 0$
$x \leq a \leq y$
$a \leq x$

$$
a \leq x
$$

$$
\begin{aligned}
& a(a-x)+\epsilon \\
& -a(a-x)+\epsilon \\
& -a(a-x)+\epsilon
\end{aligned}
$$$$
a(a-y)
$$$$
-a(a-y)
$$$$
-a(a-y)
$$$$
-a(y-a)
$$

Circular preferences are straightforward to characterize: the weight on issue 1 always equals $1 / 2$. To transform the extremist preferences above to circular preferences, simply change the coefficient of the term in ( )'s to $1 / 2$ in all cases. 


\section{A.2 Landscape Formation Proofs}

Claim A.1 Given $y \geq 0$, the measure of votes that the challenger party located at $x$ receives against an incumbent located at $y$ if voters have centrist preferences equals

$$
c(x, y, b)=\frac{6 b-3 x^{2}+\operatorname{sign}(x) \cdot x^{3}+3 y^{2}+y^{3}}{12 b}
$$

The proof consists of three parts. Let $u c(x, y$,$) equal the measure of the voters who vote$ for the challenger party if $x \geq y$..

$$
\begin{aligned}
& u c(x, y, b)=\int_{x}^{1} \int_{-b}^{(x-y)(1-a)} \frac{1}{4 b} d z d a+\int_{0}^{x} \int_{-b}^{(x+y-2 a)(a-1)} \frac{1}{4 b} d z d a \\
& \quad+i n t_{y}^{0} \int_{-b}^{(2 a-x-y)(1+a)} \frac{1}{4 b} d z d a+\int_{-1}^{y} \int_{-b}^{(y-x)(1+a)} \frac{1}{4 b} d z d a
\end{aligned}
$$

Integrating yields:

$$
u c(x, y, b)=\frac{6 b-3 x^{2}+x^{3}+3 y^{2}+y^{3}}{12 b}
$$

Let $m c(x, y, b)$ equal the measure of the voters who vote for the challenger party if $0 \leq x \leq y$.

$$
\begin{gathered}
m . c(x, y, b)=\int_{0}^{1} \int_{-b}^{(x-y)(1-a)} \frac{1}{4 b} d z d a+\int_{x}^{0} \int_{-b}^{(x-y)(a+1)} \frac{1}{4 b} d z d a \\
\quad+\int_{x}^{y} \int_{-b}^{(2 a-x-y)(1+a)} \frac{1}{4 b} d z d a+\int_{-1}^{y} \int_{-b}^{(y-x)(1+a)} \frac{1}{4 b} d z d a
\end{gathered}
$$

Integrating yielcls:

$$
m c(x, y, b)=\frac{6 b-3 x^{2}-x^{3}+3 y^{2}+y^{3}}{12 b}
$$

Finally, let $l c(x, y, b)$ equal the measure of voters who vote for the challenger party if $x \leq 0$. 


$$
\begin{gathered}
l c(x, y, b)=\int_{0}^{1} \int_{-b}^{(x-y)(1-a)} \frac{1}{4 b} d z d a+\int_{x}^{0} \int_{-b}^{(x-y)(a+1)} \frac{1}{4 b} d z d a \\
\quad+\int_{y}^{x} \int_{-b}^{(x+y-2 a)(1+a)} \frac{1}{4 b} d z d a+\int_{-1}^{x} \int_{-b}^{(y-x)(1+a)} \frac{1}{4 b} d z d a
\end{gathered}
$$

Integrating yields:

$$
l c(x, y, b)=\frac{6 b-3 x^{2}-x^{3}+3 y^{2}+y^{3}}{12 b}
$$

The result follows.

Claim A.2 Given $y \geq 0$, the measure of votes that the challenger party located at $x$ receives against an incumbent localed al y if voters have extremist preferences equals

$$
\epsilon(x, y, b)=\frac{6 b-\operatorname{sign}(x) \cdot x^{3}-y^{3}}{12 b}
$$

The proof consists of three parts. Let $u e(x, y$,$) equal the measure of the voters who vote$ for the challenger party if $x \geq y$.

$$
\begin{gathered}
u e(x, y, b)=\int_{x}^{1} \int_{-b}^{(a x-a y)} \frac{1}{4 b} d z d a+\int_{0}^{x} \int_{-b}^{(2 a-x-y)(a)} \frac{1}{4 b} d z d a \\
+\int_{y}^{0} \int_{-b}^{(x+y-2 a)(2 a)} \frac{1}{4 b} d z d a+\int_{-1}^{y} \int_{-b}^{(a x-a y)} \frac{1}{4 b} d z d a
\end{gathered}
$$

Integrating yields:

$$
u e(x, y, b)=\frac{6 b-x^{3}-y^{3}}{12 b}
$$

Let me $(x, y, b)$ equal the measure of the voters who vote for the challenger party if $0 \leq x \leq y$.

$$
m e(x, y, b)=\int_{0}^{1} \int_{-b}^{(a x-a y)} \frac{1}{4 b} d z d a+\int_{x}^{0} \int_{-b}^{(a y-a x)} \frac{1}{4 b} d z d a
$$




$$
+\int_{x}^{y} \int_{-b}^{(x+y-2 a)(a)} \frac{1}{4 b} d z d a+\int_{-1}^{y} \int_{-b}^{(a x-a y)} \frac{1}{4 b} d z d a
$$

Integrating yields:

$$
m e(x, y, b)=\frac{6 b+x^{3}-y^{3}}{12 b}
$$

Finally, let $l e(x, y, b)$ equal the measure of voters who vote for the challenger party if $x \leq 0$.

$$
\begin{gathered}
l e(x, y, b)=\int_{0}^{1} \int-b^{(a x-a y)} \frac{1}{4 b} d z d a+\int_{x}^{0} \int-b^{(a y-a x)} \frac{1}{4 b} d z d a \\
\quad+\int_{y}^{x} \int_{-b}^{(2 a-x-y)(a)} \frac{1}{4 b} d z d a+\int_{-1}^{x} \int_{-b}^{(a x-a y)} \frac{1}{4 b} d z d a
\end{gathered}
$$

Integrating yields

$$
l e(x, y, b)=\frac{6 b+x^{3}-y^{3}}{12 b}
$$

The result follows. 


\section{References}

Barry, Brian. 1970. Sociologists, Economists and Democracy. University of Chicago Press, Chicago, IL.

Campbell, Angus, Philip Converse, Warren Miller, and Donald Stokes. 1960. The American Voter. New York: John Wiley and Sons.

Davis, Otto, Melvin Hinich, and Peter Ordeshook. 1970. "An Expository Development of a Mathematical Model of the Electoral Process." American Political Science Review. 64:426- 448 .

Downs, Anthony. 1957. An Economic Theory of Democracy. New York: Harper and Row.

Fiorina, Morris. 1981. Retrospective Voting in American National Elections. New Haven: Yale University Press.

Hinich, Melvin J, and P. C. Ordeshook. 1970. "Plurality Maximization vs. Vote Maximization: A Spatial Analysis with Variable Participation." American Political Science Review. 64:722-91.

Holland, John H. 1975. Adaptation in Natural and Artificial Systems. Ann Arbor, University of Michigan Press.

Holland, John H. and John H. Miller. 1991. "Artificial Adaptive Agents and Economic Theory." American Economic Review, Papers and Proceedings. 81:365-70.

Keith, Bruce E., et al. 1992. The Myth of the Independent Voter. Berkeley: University of California Press.

Kollman, Ken, John H. Miller, and Scott E. Page. 1992. "Adaptive Parties in Spatial Elections." American Political Science Review. 86: 929-37.

Kollman, Ken, John H. Miller, and Scott E. Page. 1993. "Political Parties and Electoral Landscapes." Social science working paper \#871, California Institute of Technology.

Kollman, Ken, John H. Miller, and Scott E. Page. 1994. "A Test of a Model of Campaign Position Taking." Presented at the annual meetings of the American Political Science Association, New York.

Kramer, Gerald. 1972. "Short-Term Fluctuations in U.S. Voting Behavior, 1896-1964." American Political Science Revicw. 64:131-43.

Kramer, Gerald. 1977. "A Dynamical Model of Political Equilibrium." Journal of Economic Theory. 15:310-3-3. 
Marimon Ramon, Ellen McGrattan, and Thomas J. Sargent 1990, "Money as a Medium of Exchange in and Economy with Artificial Adaptive Agents." Journal of Economic Dynamics and Control 14: 329-373.

McKelvey, Richard D. 1976. "Intransitivities in Multidimensional Voting Models and Some Implications for Agenda Control." Journal of Economic Theory. 12:472482.

Ordeshook, Peter C. 1986. Game Theory and Political Theory. New York: Cambridge University Press.

Page, Scott E., Ken Kollman, and John H. Miller. 1992. "Adaptive Parties and Spatial Voting Theory." in Information, Participation, and Choice: An Economic Theory of Democracy in Perspective, edited by B. Grofman, The University of Michigan Press, Ann Arbor, pp 161-173.

Plott, Charles. 1967. "A Notion of Equilibrium and Its Possibility Under Majority Rule." American Economic Review. 79:787- 806.

Popkin, Samuel L. 1992. The Reasoning Voter. Chicago: University of Chicago Press.

Sears, David, et al. 1980. "Self Interest vs. Symbolic Politics in Policy Attitudes and Presidential Voting." American Political Science Review. 74:670-?? 